\title{
Spectroscopic investigation of the high-current phase of a pulsed GMAW process
}

\author{
M E Rouffet, M Wendt, G Goett, R Kozakov, H Schoepp, K D Weltmann and D Uhrlandt \\ INP-Greifswald, Leibniz Institute for Plasma Science and Technology, \\ Felix-Hausdorff-Str.2, 17489 Greifswald, Germany. \\ E-mail: schoepp@inp-greifswald.de
}

\begin{abstract}
While metal vapours have an important impact on the efficiency of the pulsed gas metal arc welding process, only a few papers are focused on this effect. In this paper, methods based on emission spectroscopy are performed to improve the understanding of the physical phenomena occurring during the high-current pulse. Boltzmann plots applied to iron lines, the Stark broadening of the $696.5 \mathrm{~nm}$ argon line and composition calculations assuming local thermodynamic equilibrium are used to determine characteristic parameters of the plasma. It is observed that the central part of the arc is mainly composed of iron. The percentage of iron increases quickly at the beginning of the high-current pulse, and slowly decreases, when the central part broadens. During the high-current phase the temperature profile has a minimum value of around $8000 \mathrm{~K}$ at the axis of the arc while the argon envelope of the central part reaches temperatures of approximately $13.000 \mathrm{~K}$. High percentage of iron and high radiation of the plasma at the centre can explain the measured shape of the temperature profile.
\end{abstract}

PACS number: $52.70 . \mathrm{Kz} 52.80 \mathrm{Mg} 81.20 . \mathrm{Vj}$

Keywords: Pulsed gas metal arc welding, GMAW, emission spectroscopy, Stark broadening Boltzmann plot

\section{Introduction}

Gas metal arc welding (GMAW) processes are widely used in industry to produce robust and high quality welded seams connecting metallic workpieces. GMAW processes use an electric arc in order to melt the welding wire so that metal droplets falling from the wire to the workpiece form the join. During the process the arc is enclosed in a shielding gas (typically argon) in order to control the current conduction path and protect the molten metal from oxidation by the atmospheric air. By using a pulsed current, it is possible to obtain a One Drop Per Pulse (ODPP) mode, which is characterized by a stable, periodical and controllable metal transfer to the workpiece [1]. The pulsed GMAW (GMAW$\mathrm{P})$ process is an effective way of achieving a controlled metal transfer to the workpiece over a wide range of heat and mass transfer rates [1]. Because of their good properties, ODPP GMAW-P processes are widely used in industry. They are intensively studied, in order to control, optimize and predict the quality of the welding seams.

The conditions needed to obtain an ODPP are well studied in literature [1-4]. Parameters 
allowing improvement of the efficiency of such process were explored by Subramanian et al. [5]. Many works investigate the metal transfer and the electrical characteristics of the ODPP GMAW-P process [6-8].

Although the radiation emitted by the metal vapour contained in the arc is an important energy loss mechanism, few works are focused on the radiation of the arc plasma, in part because of the difficulty to measure a time-dependent spectroscopic signal [9]. Zielinska et al. [10] explore the characteristic of the arc with spectroscopic methods, in a study on the influence of the shielding gas on the plasma parameters in a GMAW spraying arc. Zielinska et al. [10] use the Stark broadening of the argon line at $696.5 \mathrm{~nm}$ and the iron line at $538.3 \mathrm{~nm}$ in order to determine radial profiles of temperature and electron density. Ton et al. [11] investigate a metal inert gas welding arc and observe a temperature minimum at the arc centre. They determine the electron temperature at the centre and in the outer region of the arc, by applying the Boltzmann plot method to iron lines and analyzing the ratio of the emission of Ar II and Ar I lines, respectively. A general overview on spectroscopic diagnostics of free burning arcs and available methods for determination of temperatures and electron densities is given in [12].

The objective of this paper is to analyze the evolution of the characteristic plasma parameters in the high-current phase of a GMAW-P process where the radiative losses are the most important. A better understanding of the radiative transfer during this phase will allow a better comprehension of the physical mechanisms occurring in the plasma, and will allow to improve the efficiency of the process. To this end a detailed, time and spatially resolved spectroscopic study of the arc during the high-current phase was performed. In this study the electron density profile were determined from the Stark broadening of the argon line at $696.5 \mathrm{~nm}$. Radial temperature profiles were obtained from Boltzmann plots of iron lines at the arc centre, while they were deduced from the electron density and composition calculations in the outer region of the arc.

The text is divided into three parts. The first part presents the experimental setup, the spectrometer and the high speed camera, and describes the welding process. The diagnostic methods are described, and error estimations are performed. Results are presented in a second part, including temperature, electron number density and iron mol fraction profiles. These results are discussed in the third part.

\section{Experimental apparatus and procedure}

\subsection{Experimental apparatus}

We use a spectrometer to explore radiation emitted by the arc during the high-current phase of the GMAW-P process. Each spectroscopic measurement is completed by electrical signal and video records, to control the process. The experimental set-up (figure 1) is composed of 4 parts: the welding process, the electrical probes, the spectrometer and the high speed camera. These components are described in later paragraphs.

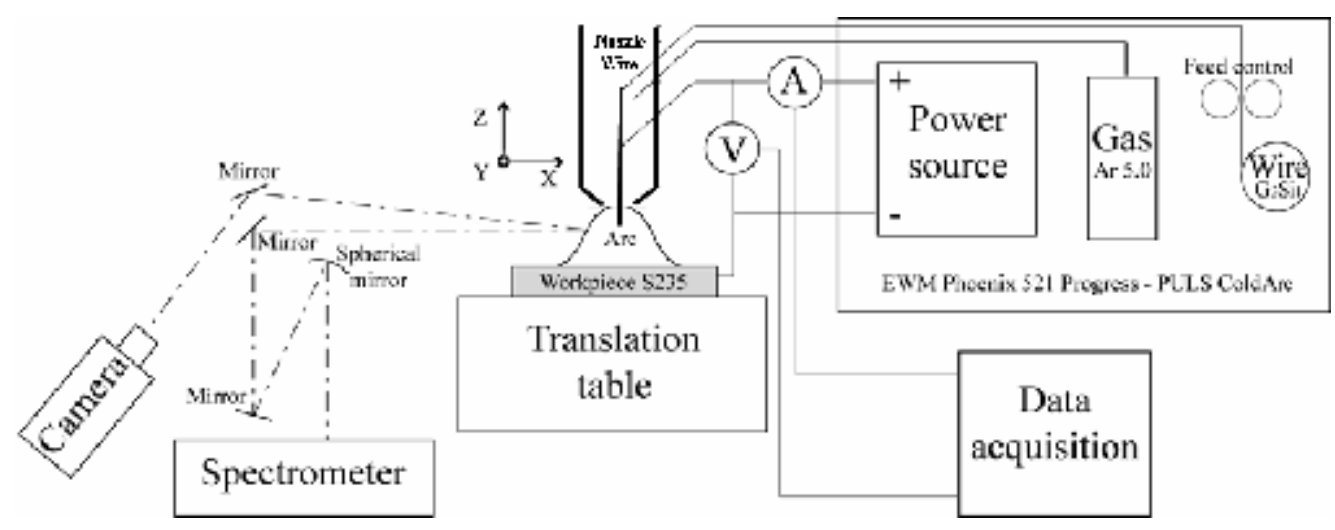

Figure 1. Experimental set-up. 


\subsubsection{The welding process}

A welding arc is generated at atmospheric pressure by a plasma torch, vertically oriented to a workpiece. Electrical power, gas flow and wire feed control were performed by a high-tech welding EWM machine Phoenix 521 Progress PULS ColdArc. The power source is operated with a positive polarity on the wire. The wire is made of steel $\mathrm{G}_{3} \mathrm{Si}_{1}$, and is $1.2 \mathrm{~mm}$ in diameter. It is fed at the velocity of $4 \mathrm{~m} / \mathrm{min}$. A $10 \mathrm{~mm}$ thick steel (S235) workpiece was employed. The chemical composition (percent by weight) of the workpiece and the wire are given in table 1 . The main component is iron, chosen because of its hardness and versatility, wear-resistance and low cost. Shielding gas is pure argon 5.0 (purity up to $99.999 \%$ ), flowing at $12 \mathrm{l} / \mathrm{min}$.

The welding wire is fed through a contact tube that is contacted to the power source. The end of the contact tube, the contact tip, is situated $6 \mathrm{~mm}$ within the nozzle and $20 \mathrm{~mm}$ above the workpiece. During the welding process, the distances between contact tip, nozzle and workpiece are kept constant in order to create arcs with reproducible plasma properties. The workpiece is moved in positive $\mathrm{x}$-direction at $30 \mathrm{~cm} / \mathrm{min}$ by means of a translation table.

Table 1. Chemical composition of the workpiece and the wire.

\begin{tabular}{cccccccccccccc} 
& Fe & C & Mn & S & P & Al & Si & Ni & Cr & Mo & V & Cu & Other \\
\hline $\mathrm{S}_{2} 25$ & $>98.3$ & $\leq 0.17$ & $\leq 1.4$ & $\leq 0.045$ & $\leq 0.045$ & $\geq 0.02$ & & & & & & & $\leq 0.009$ \\
\hline $\mathrm{G}_{3} \mathrm{Si}_{1}$ & $>96.2$ & $\leq 0.14$ & $\leq 1.6$ & 0.025 & 0.025 & 0.02 & $\leq 1.0$ & 0.15 & 0.15 & 0.15 & 0.03 & 0.35 & $\begin{array}{c}\mathrm{Ti}+\mathrm{Zr}: \\
0.15\end{array}$
\end{tabular}

The electrical measurements are performed using current and differential voltage probes. The current is measured with a Hall-Effect transducer (LB 200-S, LEM) with a response time below $1 \mu \mathrm{s}$ and an accuracy of $0.5 \%$. The voltage is measured with the high voltage differential probe (P5200, Tektronix) at the contact tip. Figure 2 shows current and voltage measured between the workpiece and the nozzle during one period, recorded by the data acquisition system (figure 1).

Figure 2 shows current and voltage curves for one duty cycle of the investigated GMAW-P. The duty cycle is divided into three phases $\mathrm{P} 1, \mathrm{P} 2$ and $\mathrm{P} 3$, during which the welding wire is molten, a molten drop propagates to the workpiece, and the plasma is sustained by a small holding current, respectively.

The pulse starts with a high-current phase (P1), where current and voltage are almost constant, at $430 \mathrm{~A}$ and about $13.2 \mathrm{~V}$, respectively. During P1, a plasma arc with strong radiation is generated between the wire and the workpiece. The current density is estimated to $300 \mathrm{~A} / \mathrm{mm}^{2}$. The heating of the wire leads to an accumulation of molten wire material at its tip. Phase P1 is chosen long enough so that electromagnetic pinching leads to the formation of a neck at the root of the molten wire material and drop detachment [6].

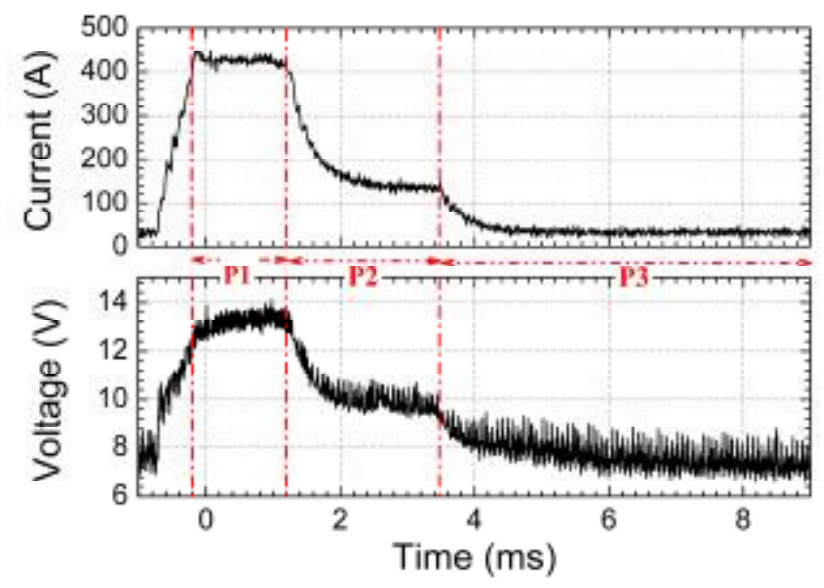

Figure 2. Current and voltage measured during one period.

During the second phase (P2), current and voltage decrease to $130 \mathrm{~A}$ and $10 \mathrm{~V}$, respectively. 
The liquid metal in the droplet continues to fall down by inertia and is guided by the axial flow of argon [13].

During a last phase (P3), current and voltage decreases further to values of $30 \mathrm{~A}$ and $7.5 \mathrm{~V}$. The plasma intensity is lowered; no spectroscopic measurements are carried out.

\subsubsection{Spectroscopy}

The plasma radiation is focused onto a $20 \mu \mathrm{m}$ wide entrance slit of a spectrometer (SpectraPro 750 , Acton research) by a spherical mirror. The optical signal is recorded side on in one propagation direction perpendicular to the arc axis, i.e. parallel to the workpiece. The radiation emitted perpendicular to the arc axis and propagating in negative X-direction is recorded as a function of the lateral position Y (cf. figure 1). The characteristics of the optical set-up allow observing the plasma radiations for distances $Y$ up to $5 \mathrm{~mm}$ from the centre of the arc. Spectra were recorded $4 \mathrm{~mm}$ above the workpiece whereas the distance between the workpiece and the nozzle was $14 \mathrm{~mm}$. The spatial resolution of the imaging system is $0.026 \mathrm{~mm} / \mathrm{pixel}$. The spectrometer is equipped with two diffraction gratings, $1200 \mathrm{~g} / \mathrm{mm}$ and $2400 \mathrm{~g} / \mathrm{mm}$ giving a wavelength resolution of 0.013 and $0.0032 \mathrm{~nm} / \mathrm{pixel}$, respectively.

We explore all the high-current phase, making measurements at four times $t_{1}, t_{2}, t_{3}$ and $t_{4}$. Each time $t_{i}$ is separated from the others by $270 \mu$ s as presented in figure 3 .

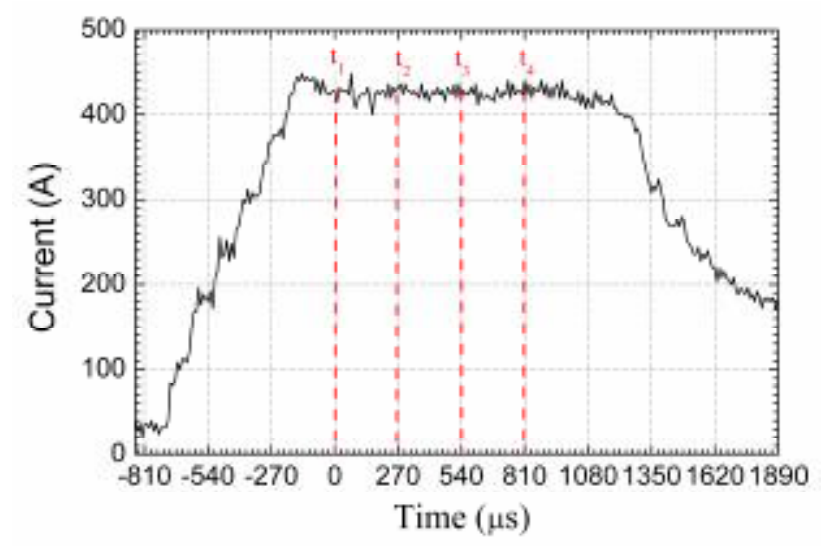

Figure 3. Definition of the four times $t_{1}, t_{2}, t_{3}$ and $t_{4}$ chosen to explore the whole high-current phase.

The spectroscopic signals are analyzed in order to calculate the temperature and electron density profiles inside the plasma. Different parts of the spectrum are successively considered, focusing on iron lines (coming from the vaporized metal) and on argon lines (coming from the shielding gas). An absolute calibration to spectral radiances in $\mathrm{W} /\left(\mathrm{nm} \mathrm{m}^{2} \mathrm{sr}\right)$ is done by means of a tungsten ribbon lamp. This lamp is imaged on the entrance slit instead of the welding arc under same optical conditions. The radiance of the $532.4 \mathrm{~nm}$ and $696.5 \mathrm{~nm}$ lines from iron and argon, respectively, are presented in the figure 4 at $t_{3}$. The values are shown as functions of the lateral $\mathrm{Y}$ position, as defined in the figure 1. In this figure the radiance is the integrated value of the separated line and represents the side-on distribution. Assuming cylindrical symmetry a polynomial fit is made before the Abel inversion could be executed. This procedure is necessary to obtain the local emissivity (in $\mathrm{W} / \mathrm{m}^{3} / \mathrm{sr}$ ) of the plasma at each radial position. A typical graph obtained is given in the figure 5 , where the local values correspond to the radiance values of the figure 4 . We note that the iron radiation is concentrated to the centre of the welding arc. The argon emissivity extends over the whole measured range of $Y$ and reaches a maximum at approximately $1 \mathrm{~mm}$ out of axis. 


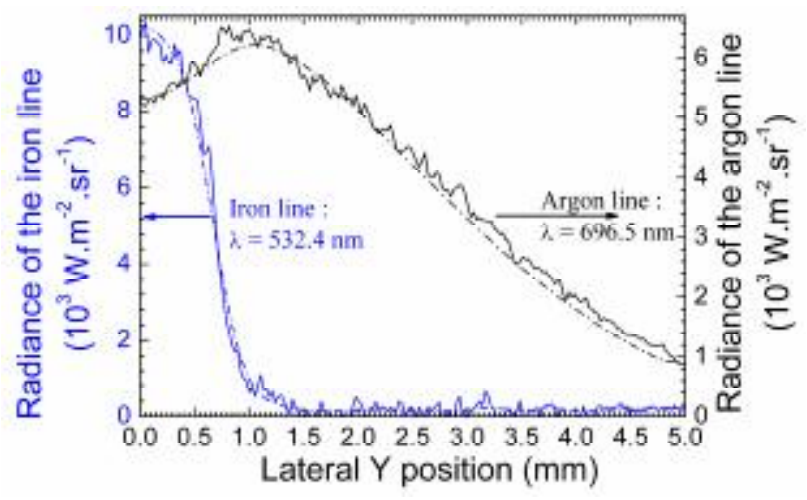

Figure 4. Radiance of the iron line at $532.4 \mathrm{~nm}$ and the argon line at $696.5 \mathrm{~nm}$ at $t_{3}=540 \mu \mathrm{s}$, as functions of the lateral Y position. Solid lines correspond to the experimental values, and dashed lines correspond to the polynomial fit values.

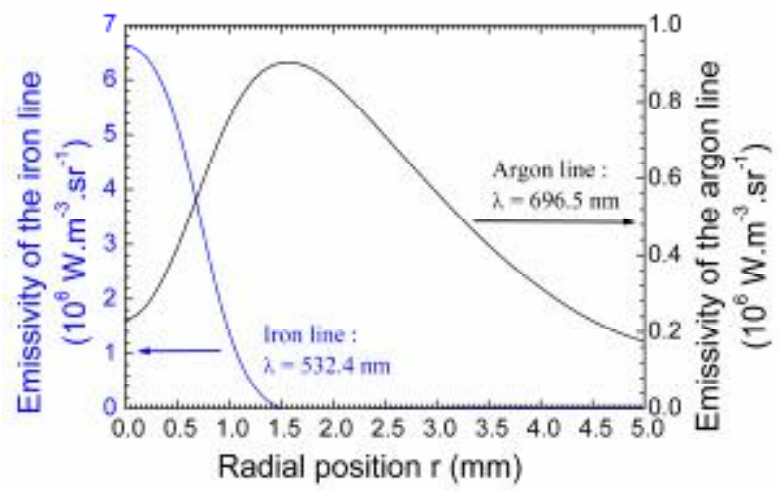

Figure 5. Emissivity of the iron line at $532.4 \mathrm{~nm}$ and the argon line at $696.5 \mathrm{~nm}$ at $t_{3}=540 \mu \mathrm{s}$, as functions of the radial position $r$, obtained by Abel inversion of the radiances presented in figure 4.

\subsubsection{High speed camera}

A high speed camera (MotionPro Y6) associated with a gray filter is used to obtain images of the plasma. The acquisition time is $4 \mu \mathrm{s}$, aperture is 32 , and the rate is $20 \mathrm{MHz}$. The acquisition of current, spectral radiance and arc images are synchronized, and a delay is adjusted to explore the pulse at different times $t_{i}$. Typical images are presented in figure 6 . The arc consists of a bright central part, corresponding to the region where the iron vapour is present, and a wider, bell shaped part showing a lower brightness. This observed shape is typical of inert gas welding arcs as for example shown in [14, 15]. During the high-current phase, the arc length is almost constant (equal to $8 \mathrm{~mm}$ ), the diameter of the central region is increasing (from $1 \mathrm{~mm}$ to $2 \mathrm{~mm}$ ), and the central part is rotationally symmetric in good approximate.

Closer inspection of the high speed images reveals that the brightness of the arc core has a maximum near the wire tip and decreases towards the workpiece. Also, in the region with high brightness, the arc core diameter has a flat maximum and decreases slowly towards the workpiece. At distances to the workpiece below approximately $2 \mathrm{~mm}$ the stagnation zone of the flow leads to a strong increase of the core diameter. The axial position of the spectroscopic measurements at $4 \mathrm{~mm}$ is chosen well above the stagnation zone, and in a region with only a small axial variation of the arc core's brightness and diameter. The modelling results given in [15] suggest that measurements closer to the wire tip would give higher plasma temperatures. 


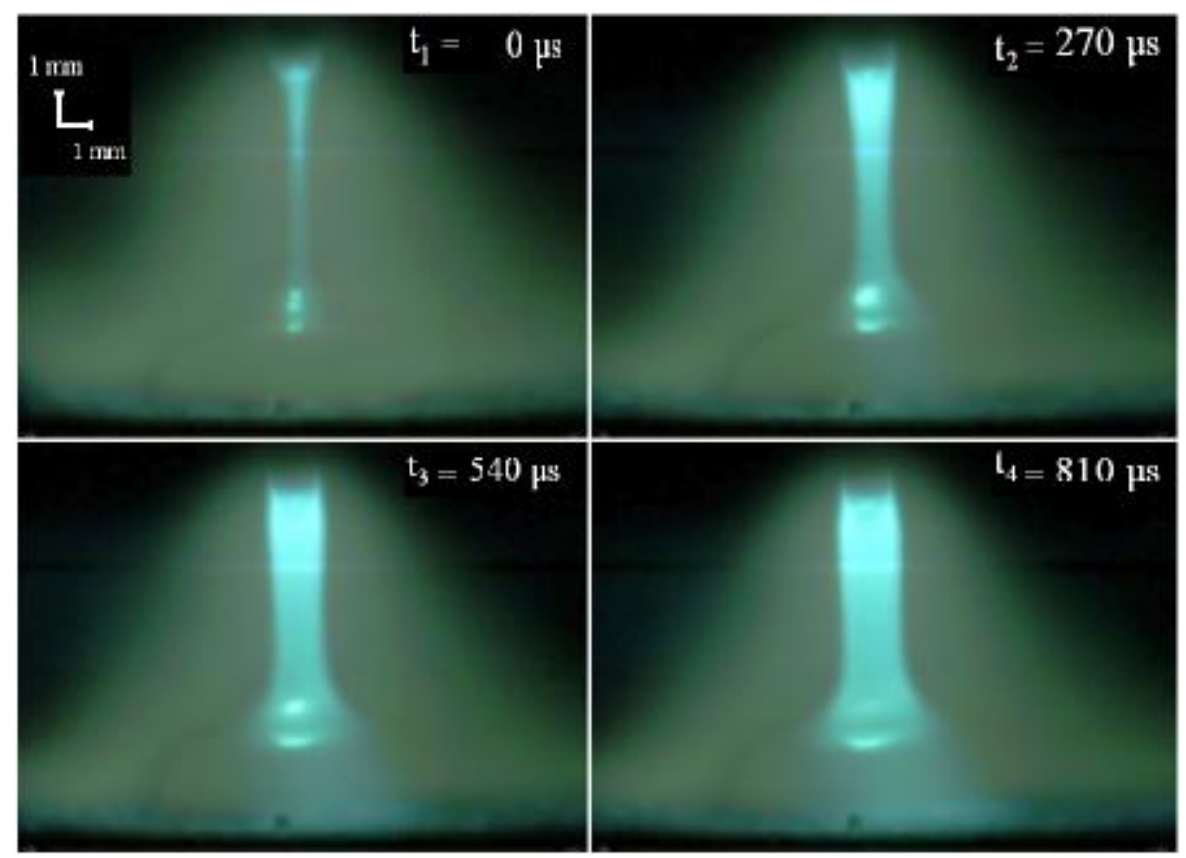

Figure 6. Pictures of the plasma at different times $t_{1}, t_{2}, t_{3}$ and $t_{4}$.

\subsection{Diagnostic methods}

Emission spectroscopy allows the determination of the plasma characteristics when a Partial Local Thermodynamic Equilibrium (PLTE) exists inside the plasma. The excitation temperature of the excited atomic levels is then equal to the electron temperature and their densities are related to the next ion's ground state population density by Saha-Boltzmann relations $[10,16]$. Following the Boltzmann plot method, we analyze iron lines to deduce the plasma temperature. The electron number density is determined using the Stark broadening of the $696.5 \mathrm{~nm}$ argon line. PLTE is not necessary for the latter method. The percentage of iron inside the plasma is deduced from both latter values and calculated plasma compositions. The applied methods are described in the next paragraphs.

\subsubsection{Plasma temperature}

In PLTE, the population of the atomic energy states adheres to a Boltzmann distribution, uniquely characterized by their temperature, $\mathrm{T}$. This temperature may be obtained in applying the Boltzmann plot diagnostic method. This method is based on the fact that the emissivities of spectral lines belonging to the same atom are connected by the relation:

$$
\ln \left(\frac{\varepsilon_{i j} \lambda_{i j}}{g_{j} A_{j i}}\right)=-\left(\frac{E_{j}}{k_{B} T}\right)+C
$$

The left-hand term is usually called Boltzmann function. The quantities $\varepsilon_{\mathrm{ij}}, \lambda_{\mathrm{ij}}$ and $\mathrm{A}_{\mathrm{ji}}$ are the emissivity $\left(\mathrm{W} / \mathrm{m}^{3} / \mathrm{sr}\right)$, the wavelength $(\mathrm{m})$, and the transition probability $\left(\mathrm{s}^{-1}\right)$ of the line corresponding to the transition from upper level $j$ to lower level $i$, respectively. $E_{j}$ and $g_{j}$ are the energy $(e V)$ and the statistical weight of level $\mathrm{j}$, respectively. $\mathrm{k}_{\mathrm{B}}$ is the Boltzmann constant. $\mathrm{T}$ is the temperature of the plasma $(\mathrm{K})$, and $\mathrm{C}$ is a constant at fixed temperature. A linear fit of $\ln \left(\varepsilon_{\mathrm{ij}} \lambda_{\mathrm{ij}} / \mathrm{g}_{\mathrm{j}} \mathrm{A}_{\mathrm{jij}}\right)$ on $\mathrm{E}_{\mathrm{j}}$ allows to deduce the plasma temperature. Seven iron lines are selected because of the large differences of their upper energy levels $E_{j}$ (table 2), and because of their close proximity so they can be measured simultaneously.

Figure 7 shows an example of a Boltzmann plot obtained at the centre of the arc at $t_{3}$. The linear fit is made by the least squares method [17]. Each point corresponds to the Boltzmann function value of one line. In this example, the slope is $-1.50047 \mathrm{eV}^{-1}$, corresponding to the temperature 
$\mathrm{T}=7734 \mathrm{~K}$

Table 2 : Characteristic of the iron lines selected for the Boltzmann diagnostic method [17].

\begin{tabular}{cccccc} 
Wavelength (nm) & $\mathbf{A}_{\mathbf{j i}}\left(\mathbf{1 0}^{\mathbf{6}} \mathbf{s}^{\mathbf{- 1}}\right)$ & Accuracy on $\mathbf{A}_{\mathbf{j i}}$ & $\mathbf{E}_{\mathbf{i}}(\mathbf{e V})$ & $\mathbf{E}_{\mathbf{j}}(\mathbf{e V})$ & $\mathbf{g}_{\mathbf{j}}$ \\
\hline 530.2299 & 6.30 & $\leq 18 \%$ & 3.2830240 & 5.6206837 & 5 \\
\hline 532.4178 & 15.00 & $\leq 18 \%$ & 3.2111889 & 5.5392422 & 9 \\
\hline 532.8038 & 1.15 & $\leq 7 \%$ & 0.9146016 & 3.2409687 & 7 \\
\hline 537.1489 & 1.05 & $\leq 7 \%$ & 0.9581568 & 3.2657053 & 5 \\
\hline 538.3369 & 56.00 & $\leq 18 \%$ & 4.3124697 & 6.6149260 & 13 \\
\hline 539.3167 & 3.10 & $\leq 18 \%$ & 3.2409687 & 5.5392422 & 9 \\
\hline 539.7127 & 2.59 & $\leq 7 \%$ & 0.9146016 & 3.2111889 & 9
\end{tabular}

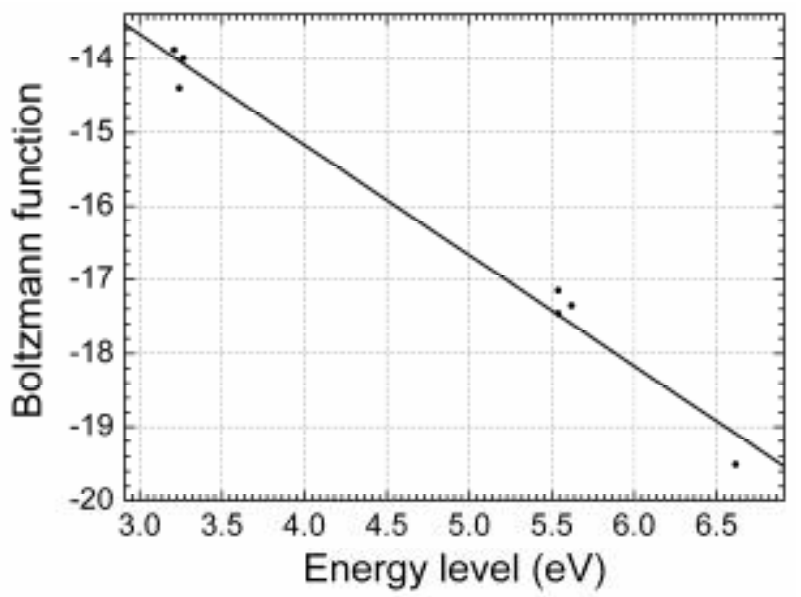

Figure 7. Example of a Boltzmann plot allowing the deduction of the plasma temperature, obtains at $t_{3}$ $=540 \mu \mathrm{s}$, at the centre of the welding arc.

\subsubsection{Electron number densities}

The electron number density in the arc is determined by analyzing the broadening of the neutral argon line at $695.5 \mathrm{~nm}$. At atmospheric pressure and temperatures usually found in GMAW-P (around $10000 \mathrm{~K}$ ) this line is mainly by electrons Stark broadened showing a Lorentz line profile with a full width at half maximum (FWHM) of approximately $0.1 \mathrm{~nm}$. The FWHM of Doppler broadening width is approximately $0.001 \mathrm{~nm}$. Additionally, the selected line shows low self-absorption, strong intensity and sufficient isolation from neighbouring lines.

Electron Stark broadening of spectral lines is caused by the interaction of the radiator with the electrons of the surrounding plasma leading to a perturbation of the lines upper and lower levels [18]. The corresponding line width is proportional to the electron density $N_{\mathrm{e}}$ and has weak temperature dependence, so that measured widths can be used to deduce the electron density.

Figure 8 presents a summary of Stark widths given in [18]. The values are reported for plasma at atmospheric pressure and at a temperature of $10^{4} \mathrm{~K}$. A fit of the FWHM $\Delta \lambda_{S}$ on the electron number density $N_{\mathrm{e}}$ in $\mathrm{m}^{-3}$ gives the relation

$$
\Delta \lambda_{S}=\lambda_{S}^{0}\left(N_{e} / 10^{23} \mathrm{~m}^{-3}\right)^{0.97762}
$$

with $\lambda_{S}^{0}=0.0803 \mathrm{~nm}$. This equation is used to determine electron number densities from measured line widths. For each lateral position $\mathrm{Y}$ the line width $\Delta \lambda_{\mathrm{s}}$ is determined by fitting a Lorentz function to 
the measured spectral radiance. An example for $\mathrm{Y}=0$, giving a width of $\Delta \lambda_{\mathrm{s}}=0.11594 \mathrm{~nm}$ which corresponds to $\mathrm{N}_{\mathrm{e}}=1.08 \times 10^{23} \mathrm{~m}^{-3}$ is shown in figure 9 .

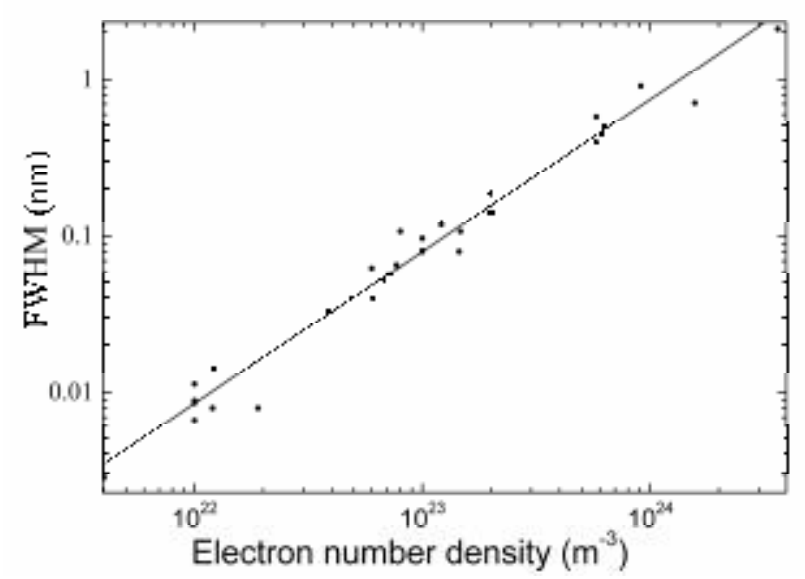

Figure 8. FWHM of the $696.5 \mathrm{~nm}$ argon line as function of electron number density. Circles correspond to literature values [18], and solid line corresponds to the linear fit.

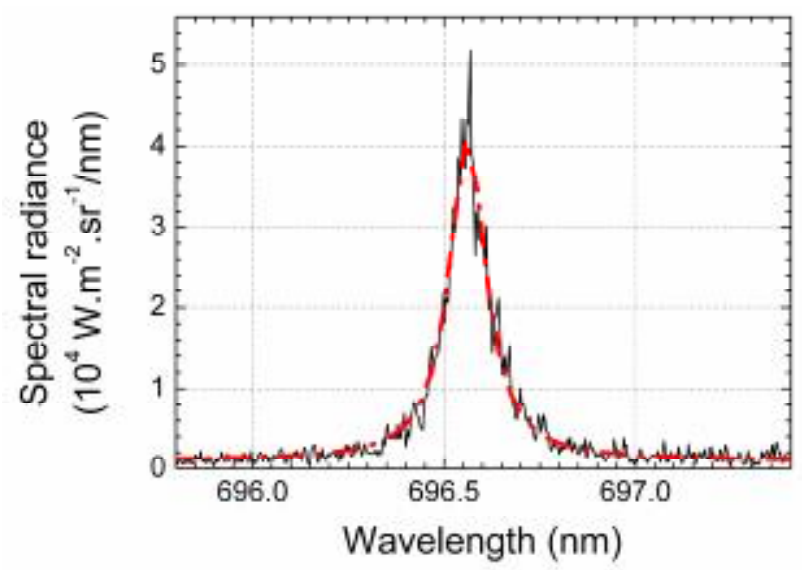

Figure 9. Lorentz line fit of the $696.5 \mathrm{~nm}$ argon line at $t=540 \mu \mathrm{s}$ and lateral position $Y=0$. Solid line corresponds to the experimental data, and dash line corresponds to the Lorentz profile fit.

\subsubsection{Composition of the plasma}

In PLTE, plasma composition can be deduced from both the plasma temperature and the electron number density, due to equilibrium equations. Calculations were made in solving a system of mass action laws together with equations for charge neutrality, given total pressure and iron content:

$$
f_{\mathrm{Fe}}=\left(\frac{N_{\mathrm{Fe}}+N_{\mathrm{Fe}^{+}}+N_{\mathrm{Fe}^{2+}}}{N_{\mathrm{Fe}}+N_{\mathrm{Fe}^{+}}+N_{\mathrm{Fe}^{2+}}+N_{\mathrm{Ar}}+N_{\mathrm{Ar} r^{+}}+N_{\mathrm{Ar}{ }^{2+}}}\right)
$$

Deviations from ideal gas behaviour due to the Coulomb interaction were taken into account following Ebeling et al. [19]. These are contributions to the total pressure and to the chemical potentials of the species, where the latter lead to an effective reduction ionization potential of the species. Also following Ebeling et al., the Planck-Larkin partition function was used for the chemical potentials evaluation. The internal energy levels of the species were taken from Moore [20] and NIST [21].

Because both $\mathrm{N}_{\mathrm{e}}$ and $\mathrm{T}$ are necessary to deduce the content of iron inside the plasma, and because $\mathrm{T}$ is only known at the centre (due to the too weak metal vapour radiation signal recorded at the edge), centre and edge are processed using two different methods.

At the centre, where both parameters $\mathrm{N}_{\mathrm{e}}$ and $\mathrm{T}$ are known, the percentage of iron atom $(\% \mathrm{Fe})$ is deduced from equilibrium equations.

At the edge of the welding arc, only $\mathrm{N}_{\mathrm{e}}$ is available. However, knowing $\% \mathrm{Fe}$ at the centre, and knowing that $\% \mathrm{Fe}$ is negligible at the edge of the plasma, it is possible to estimate the probable iron 
mol fraction profile at the boundary between the centre and the edge. It allows to know both parameters $\mathrm{N}_{\mathrm{e}}$ and $\% \mathrm{Fe}$. T is then deduced from equilibrium equations.

The table 3 summarizes the different tactics adopted to deduce the third parameter from both others.

Table 3. Summary describing the way to deduce the third parameter from both others.

\begin{tabular}{cccc} 
Position in the arc & Calculated values & Probable values & Deduced values \\
\hline Centre $(0 \mathrm{~mm}<\mathrm{r} \leq 1 \mathrm{~mm})$ & $\mathrm{N}_{\mathrm{e}}, \mathrm{T}$ & $\% \mathrm{Fe}$ \\
\hline Edge $(\mathrm{r}>1 \mathrm{~mm})$ & $\mathrm{N}_{\mathrm{e}}$ & $\% \mathrm{Fe}$ & $\mathrm{T}$
\end{tabular}

\subsubsection{Error estimation}

Iron and argon lines are not recorded simultaneously. Consequently, electron number density and plasma temperature are measured at two different current pulses. A first error source is to associate $\mathrm{N}_{\mathrm{e}}$ at $\mathrm{T}$, whereas the state of the plasma is not exactly the same. To take into account this error, the variation of the parameters temperature, electron number density and percentage of iron at the same time in the period but over several pulses has been estimated. Such variations are lower than the error bars estimated below, whatever the parameter considered. The state of the plasma is almost the same whatever the current pulse considered. The error induced by variations of plasma state at various current pulses is then neglected. In the next paragraphs, estimation of other errors is made, focusing on each diagnostic method.

Boltzmann plot method. The error of the temperature resulting from the Boltzmann plot is:

$$
\frac{d T}{T}=\frac{k_{B} T}{\left[\sum_{l=1}^{7} E_{l}^{2}-\frac{1}{7}\left(\sum_{l=1}^{7} E_{l}\right)^{2}\right]^{1 / 2}} \times\left(\frac{d \varepsilon_{i j}}{\varepsilon_{i j}}+\frac{d A_{j i}}{A_{j i}}\right)
$$

The first term takes into account the upper energy level $E_{1}$ of the seven lines selected to apply the Boltzmann plot method. The left-hand term in the parenthesis corresponds to the error of emissivity values resulting here from limited measurement reproducibility. This error is $9 \%$ at the centre and increases up to $15 \%$ at a radial position of around $1 \mathrm{~mm}$. The Abel inversion introduces an error on $\varepsilon_{\mathrm{ij}}$ due to the smoothing of the radiances. This error is estimated to $15 \%$. The second term in the parentheses corresponds to the error generated by the uncertainty of the transition probability $\mathrm{A}_{\mathrm{ji}}$ taken from the NIST compilation [21] (see accuracy in table 2). The error of $\mathrm{A}_{\mathrm{ji}}$ is considered equal to $18 \%$, giving an upper limit of the error generated. Applying equation 4, a temperature error of $8 \%$ at the centre of the plasma, and $9 \%$ at $1 \mathrm{~mm}$ radius is obtained. Using more refined numerical methods for Abel inversion may reduce the total error of the temperature.

Stark broadening method. Equation 2 leads to an error estimation of the electron density of

$$
\frac{\Delta N_{e}}{N_{e}}=1.002\left(\frac{\Delta\left(\Delta \lambda_{S}\right)}{\Delta \lambda_{S}}+\frac{\Delta \lambda_{S}^{0}}{\lambda_{S}^{0}}\right) .
$$

The first term (on the right hand side of equation 5) takes into account the uncertainty of the line width due to the fitting procedure and due to the random error caused by fluctuations of the arc plasma. It is estimated to $3.5 \%$. The second term is caused by the uncertainty of the scaling constant $\lambda_{s}^{0}$. Zielinska et al. [10] use a scaling constant of $0.0739 \mathrm{~nm}$, while Dimitrijevic et al. [18] give a Stark width of $0.0637 \mathrm{~nm}$ for a temperature of $10000 \mathrm{~K}$ and an electron density of $10^{23} \mathrm{~m}^{-3}$. Using these three values an uncertainty of $12 \%$ can be estimated for $\lambda_{s}^{0}$. Zielinska et al. [10] use an additional temperature dependence of $\Delta \lambda_{s}$ proportional to $\mathrm{T}^{0.3685}$. This would give an additional term $0.3685 \Delta \mathrm{T} / \mathrm{T}$ on the right hand side of eq. 5 and an additional error of $3.6 \%$. Thus, the resulting total error of the electron density is less than $20 \%$. While the error of the electron density is limited by the uncertainty of $\lambda_{S}^{0}$ 
the uncertainty of $\Delta \lambda_{S}$ may be reduced by using spectrometers with a higher signal to noise ratio.

Deduction of the third parameter using equilibrium equations. The use of equilibrium equations is justified if the plasma is in LTE. Deviation from LTE is a source of error, especially at the edge of the plasma. The studie given in [10] indicates that LTE is closely fulfilled in the GMAW process so that errors due to deviations from LTE are considered negligibly small.

At the arc centre, considering an error of $8 \%$ on the temperature determined by Boltzmann plot method, and an error of $18 \%$ on the electron number density deduced from Stark broadening, the error on the percentage of iron is estimated to $50 \%$.

At the edge of the arc core, considering an error of $18 \%$ on the electron number density deduced from Stark broadening, and an error of $50 \%$ on the percentage of iron, leads to an error of $8 \%$ of the plasma temperature. The error of the temperature is relatively small due to the rapid increase of the electron density with rising temperature.

Table 4. Summary concerning the uncertainties of diagnostic methods.

\begin{tabular}{|c|c|c|}
\hline Parameters & Diagnostic method & Accuracy \\
\hline $\mathrm{T}$ & Boltzmann plot & $\begin{array}{c}8 \% \text { at } \mathrm{r}=0 \mathrm{~mm} \\
25 \% \text { at } \mathrm{r}=1.5 \mathrm{~mm}\end{array}$ \\
\hline $\mathrm{N}_{\mathrm{e}}$ & Stark broadening & $18 \%$ \\
\hline$\% \mathrm{Fe}$ & Composition computation & $50 \%$ \\
\hline
\end{tabular}

\section{Results}

Figure 10 presents temperature profiles of the plasma at different times $t_{i}$ of the high-current phase of the process. For all four times the temperature is lower at the centre of the plasma, as expected and observed in previous experiments [10-11] and a numerical model [15]. This inner temperature, around $8000 \mathrm{~K}$, is stable all along the high-current phase. Temperature increases rapidly at around $1 \mathrm{~mm}$, and then decreases slowly towards the edge of the arc. The radius of the inner (cold) region of the arc expands from $1.2 \mathrm{~mm}$ at $\mathrm{t}=0$ to around $1.7 \mathrm{~mm}$ at $\mathrm{t}=810 \mu \mathrm{s}$. During this expansion both the temperature at the arc centre and the temperature profile in the outer region remain approximately constant.

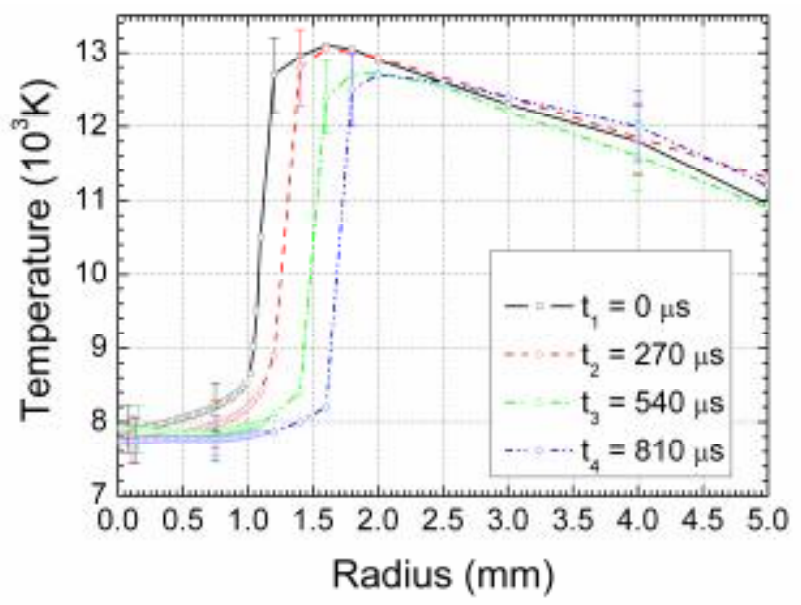

Figure 10. Temperature profiles of the plasma at different time $t_{i}$ of the high-current phase.

Figure 11 shows the electron density of the welding arc in dependence of the radial position, for the four times $t_{i}$ of the high-current phase. The variation over time is low in comparison with the error bars estimated. Nevertheless, first conclusions on the spatial and temporal behaviours can be given. The electron density is weakly lower at the centre of the plasma than at the radius of $0.6 \mathrm{~mm}$. 
After this distance, values decreases slowly. The radial position of the maximum value of $\mathrm{N}_{\mathrm{e}}$ increases slightly with time along the high-current phase.

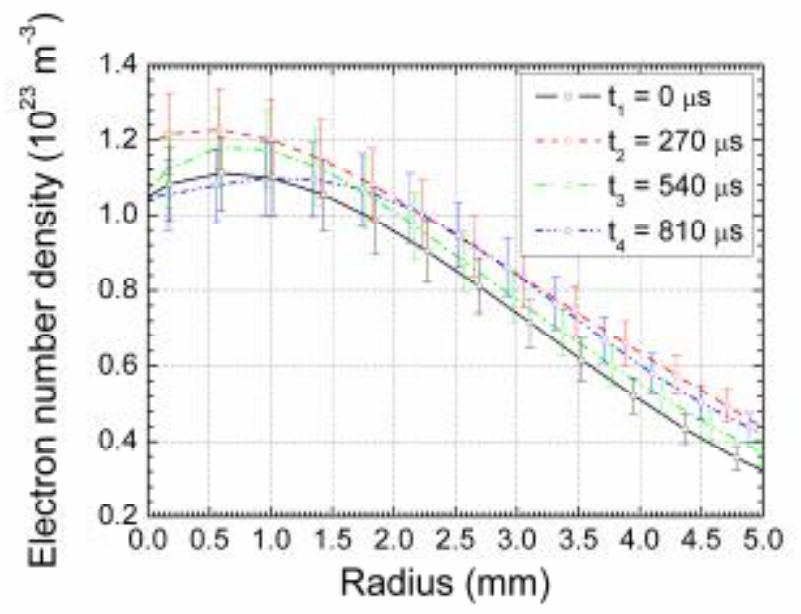

Figure 11. Evolution of the electron density at different times $t_{i}$ of the high-current pulse.

Figure 12 presents the evolution of the iron atom percentage in the plasma, in function of the radial position, at the four different times of the high-current phase. The iron vapour density achieves a maximum value at the centre of the arc, and decreases strongly at around $1 \mathrm{~mm}$ radius. For radial positions larger than $3 \mathrm{~mm}$, the plasma can be considered as pure argon plasma. Percentage of iron at the centre of the arc increases from $50 \%$ to $70 \%$ at the beginning of the high-current phase. Then it continuously decreases until the end of the high-current phase.

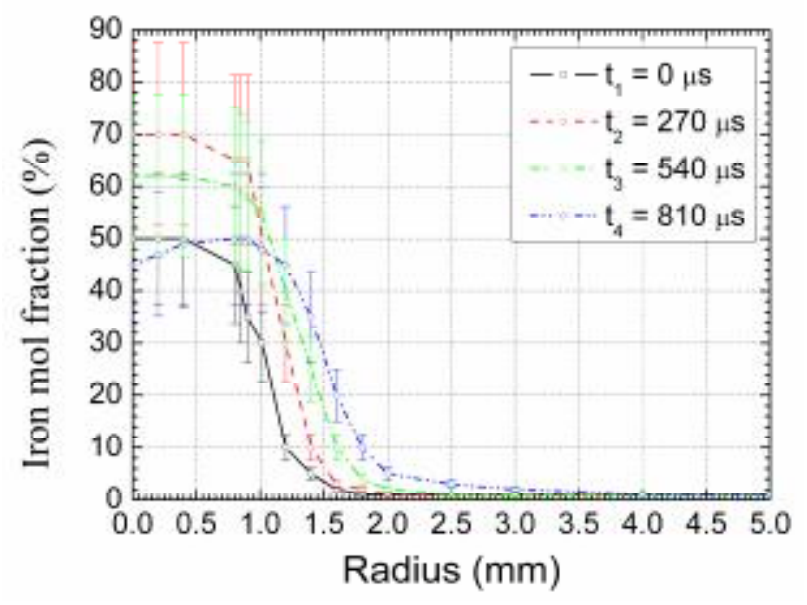

Figure 12. Iron mol fraction at different times $t_{i}$ of the high-current phase.

\section{Discussion}

During the whole high-current phase, metal vapours coming from the wire are introduced into the plasma. Due to the flow of the shielding gas, these vapours are concentrated at the centre of the arc.

The temperature profile of stationary arcs is determined by the energy balance:

$$
\rho c_{P} v_{z} \frac{\partial T}{\partial z}=\sigma E^{2}-4 \pi \varepsilon_{N}+\frac{1}{r} \frac{\partial}{\partial r}\left(r \kappa \frac{\partial T}{\partial r}\right)
$$

Here $\rho, c_{P}, v_{z}, \varepsilon_{N}$ and $\kappa$ are the mass density, specific heat capacity, the axial flow velocity, the net emission and the heat conductivity, respectively. Considering that the convective term on the left hand side of (6) is small with respect to the source terms, the pronounced minimum of the temperature profile at the arc axis can be understood following the arguments of Lowke et al. [15]. In pure argon 
arcs where the net emission is small the ohmic heating term is balanced by the heat conduction term, so that heating leads to a negative curvature of the temperature profile. Consequently, the temperature profile has a maximum on the arc axis. For significant amounts of iron the net emission of the plasma is several orders of magnitude higher than for pure argon, e.g. for $8000 \mathrm{~K}$ the emission of iron is $7 \times 10^{10} \mathrm{~W} / \mathrm{m}^{3} / \mathrm{sr}$ while it is only $1 \times 10^{7} \mathrm{~W} / \mathrm{m}^{3} / \mathrm{sr}$ for pure argon [22]. In this case the sum of heating and net emission can become negative, so that the curvature of the temperature gets positive, thus leading to a temperature minimum on the arc axis. At the radial position where the curvature of the temperature vanishes ohmic heating is balanced by the net emission. Comparison of figures 10 and 12 shows that this is the case for an iron content of approximately $20 \%$.

Figure 10 shows a radial expansion of the arc core with a velocity of approximately $0.6 \mathrm{~m} / \mathrm{s}$. This expansion may be due to radial diffusion or an increase of the evaporation region on the welding wire. The decrease of the iron content towards the end of the high-current phase can be caused by the expansion of the arc core but also by a decrease of the evaporation rate of the welding wire.

Haidar investigated the population of the argon ground state in a TIG welding arc using a numerical non-equilibrium model [16]. For distances from the tungsten cathode bigger than $2.5 \mathrm{~mm}$ the deviation from LTE was small. The deviation in the model is caused by the divergence of plasma flow. In regions where the flow is only in axial direction and its variations only in radial direction this term equals zero and LTE is assumed. At the position of the measurements made here the arc appears homogeneous in z-direction so that we do not expect deviations from LTE due to the flow. However, near the wire tip the divergence of the flow may lead to deviations from LTE. In argon arcs the overpopulation of the ground state is enabled by a large energy separation of the first excited state from the ground state $(11.55 \mathrm{eV})$. For iron this separation is much smaller $(0.86 \mathrm{eV}$ for the lowest level not in the $\mathrm{a}^{5} \mathrm{D}$ Term) so that an overpopulation of the ground state is less likely.

Very recently, results of fluid simulations $[23,24]$ for GMAW processes similar to the object of this work have been reported. A temperature minimum in the arc axis has been found in the both theoretical studies too. In [23] the influence of a different extent of metal evaporation has been studied, however transport data for pure argon have been applied for this part of the study. Material properties of the mixture of argon and iron vapour including diffusion effects have been used in the simulations in [24]. In addition, comparative calculations applying each one property of iron in the work [24] have been revealed that the increased power loss due to radiation represents the main reason for the low temperature of the iron vapour core. The absolute values of the temperature in these studies can not be compared with the measured one because the considered processes are not exactly the same. A direct comparison is planned for future work.

\section{Conclusion}

A spectroscopic study was performed at the high-current phase of a GMAW-P process. Plasma characteristics have been determined utilizing Boltzmann plot analysis of iron line radiation, Stark broadening analysis of an argon line and plasma composition calculations.

The diagnostics allowed a sufficiently high accurate determination of the plasma temperature at least in the arc core as well as estimations of the radial and temporal behaviour of electron density and iron mol fraction. The centre of the arc is mainly composed of iron, coming from the wire, where the plasma temperature is around $8000 \mathrm{~K}$ over the whole high-current phase. The percentage of iron increases considerably at the beginning of the current pulse. Later on in the high-current phase it slowly decreases together with a radial expansion of the region dominated by the iron vapour. The metal vapour core shows a sharp edge where the temperature increases radially up to $13000 \mathrm{~K}$ to compensate the lower ionization and as a result of the lower radiative losses of the shielding gas argon.

Beside the first discussion of the main impact of iron vapour in the welding arc, this work should be the base of further studies like the impact of shielding gas kind and flow or the analysis of arc regions near the wire and the workpiece. In addition, the use of the experimental results for validations of corresponding arc models is aimed in future studies.

\section{Bibliography}


Wang G, Huang P G and Zhang Y M 2004 Numerical analysis of metal transfer in gas metal arc welding under modified pulsed current conditions, Metallurgical and materials transactions B 35B 857.

Jacobsen N 1992 Monopulse investigation of drop detachment in pulsed gas metal arc welding, J. Phys. D: Appl. Phys. 25 783-797.

Arif N, Hak Lee J and Don Yoo C 2009 Force-displacement model for analysis of pulsed-GMAW, J. Phys. D: Appl. Phys. 42035504 (7pp).

Zhang Y M, Liguo E and Kovacevic R 1998 Active metal transfer control by monitoring excited droplet oscillation, Welding research supplement 388s-395s.

Subramaniam S, White D R, Jones J E and Lyons D W 1999 Experimental approach to selection of pulsing parameters in pulsed GMAW, Welding research supplement 166s172 s.

Choi S K, Yoo C D and Kim Y S 1998 The dynamic analysis of metal transfer in pulsed current gas metal arc welding, J. Phys. D: Appl. Phys. 31 207-215.

Praveen P, Kang M J and Yarlagadda P K D V 2006 Arc voltage behavior of one drop per pulse mode in GMAW-P, Journal of achievements in materials and manufacturing engineering 17 389-392.

Hu J and Tsai H L 2006 Effects of current on droplet generation and arc plasma in gas metal arc welding, Journal of applied physics $\mathbf{1 0 0} 053304$.

Pellerin N, Zielinska S, Pellerin S, Valensi F, Musiol K, de Izarra C, Briand F and Richard F 2006 Experimental investigations of the arc MIG-MAG welding, AIP Conference proceeding $\mathbf{8 1 2} 80-87$.

Zielinska S, Musiol K, Dzierzega K, Pellerin S, Valensi F, de Izarra C and Briand F 2007 Investigations of GMAW plasma by optical emission spectroscopy, Plasma Sources Sci. Technol. 16 832-838.

Ton H 1975 Physical properties of the plasma-MIG welding arc, J. Phys. D: Appl. Phys. 8 922-933.

Wiese W L 1991 Spectroscopic diagnostics of low temperature plasmas: techniques and required data, Spectrochimica Acta 46B 831-841.

Lowke J J, Kovitya P and Schmidt H P 1992 Theory of free-burning arc columns including the influence of the cathode, J. Phys. D: Appl. Phys. 25 1600-1606.

Haidar J 1998 An analysis of the formation of metal droplets in arc welding, J. Phys. D: Appl. Phys. 31 1233-1244.

Lowke J J, Tanaka M and Murphy A B 2009 Metal vapours in MIG arcs cause (1) minima in central arc temperatures and (2) increased arc voltages, Singapore doc. 2121139-09 1-9.

Haidar J 1997 Departure from local thermodynamic equilibrium in high-current free burning arcs in argon, J. Phys. D: Appl. Phys. 30 2737-2943.

Van Huel S and Vandewalle J 1991 The Total Least Squares Problem. SIAM, 


\section{Philadelphia, PA.}

[18] Dimitrijevic M S, Christova M and Sahal-Brechot S 2007 Stark broadening of visible Ar I spectral lines, Phys. Scr. 75 809-819.

[19] Ebeling W, Kraeft W D and Kremp D 1976 Theory of bound states and ionization equilibrium in Ergebnisse der Plasmaphysik und der Gaselektronik Vol 5, eds R. Rompe, M. Steenbek (Akademie-Verlag : Berlin).

[20] Moore C E 1948 Atomic energy levels, Circular 467 (National bureau of standards: Washington).

[21] Ralchenko Y, Kramida A E, Reader J and NIST ASD Team 2007 NIST Atomic Spectra Database (version 3.1.3), available on line at http://physics.nist.gov/PhysRefData/, National Institute of Standards and Technology, Gaithersburg, MD.

[22] Menart J and Malik S 2002 Net emission coefficients for argon-iron thermal plasmas, J. Phys. D: Appl. Phys. 35 867-874.

[23] Haidar J 2010 The dynamic effects of metal vapour in gas metal arc welding, JPhys. D: Appl. Phys. 43165204

[24] Schnick M, Füssel U, Hertel M, Spille-Kohoff A, and Murphy A B 2010, Metal vapour causes a central minimum in arc temperature in gas-metal arc welding through increased radiative emission, J. Phys. D: Appl. Phys. 43022001 